Relations industrielles

Industrial Relations

\title{
La déclaration de principes de la C.T.C.C
}

Volume 10, numéro 1, décembre 1954

URI : https://id.erudit.org/iderudit/1022791ar

DOI : https://doi.org/10.7202/1022791ar

Aller au sommaire du numéro

Éditeur(s)

Département des relations industrielles de l’Université Laval

ISSN

0034-379X (imprimé)

1703-8138 (numérique)

Découvrir la revue

Citer ce document

(1954). La déclaration de principes de la C.T.C.C. Relations industrielles /

Industrial Relations, 10(1), 56-60. https://doi.org/10.7202/1022791ar

Tous droits réservés @ Département des relations industrielles de l’Université Laval, 1954
Ce document est protégé par la loi sur le droit d'auteur. L’utilisation des services d'Érudit (y compris la reproduction) est assujettie à sa politique d'utilisation que vous pouvez consulter en ligne.

https://apropos.erudit.org/fr/usagers/politique-dutilisation/ 
abstraite, mais leur forme concrète dépend aussi des circonstances de temps, de lieu et de culture.

4. Un dernier point d'orientation morale que Nous voudrions indiquer est le contrôle moral du succès.

Il ne s'agit pas ici d'une «morale du succès » qui tire sa valeur et sa justification du succès matériel obtenu sous la forme d'un gain considérable. Le manque de conscience peut aussi conduire à de gros profits et reste cependant un manque de conscience.

Mais les succès moraux et les effets d'un système en fournissent une vérification. Celle-ci s'appuie sur la conviction qu'on peut reconnaître aux fruits d'un arbre s'il vaut quelque chose ou s'il ne vaut rien. Le bon arbre ne peut porter de mauvais fruits, ni le mauvais de bons fruits.

Dans la vie des affaires, beaucoup de manipulations sont si compliquées et il est au début si difficile d'y voir clair, que le temps seul permet de se rendre compte si le chemin suivi est moral et licite ou non, s il concorde avec le vrai bien de l'individu et de la communauté ou s'il s'y oppose. Il est très important ici de savoir jeter un regard en arrière. Le contrôle du succès et le jugement de valeur qui s'ensuit seront souvent plus sûrs et plus complets dans une délibération commune que si chacun s'appuie uniquement sur son jugement propre.

Voilà ce que Nous voulions vous dire au sujet de cet aspect de votre activité. Toute contribution que vous pourrez apporter, Messieurs, à une conception plus noble et plus désintéressée de votre profession et de la vie des affaires aura pour effet de favoriser la restauration morale, que Nous appelons avec vous de Nos voeux les plus ardents. A cette intention, et pour attirer sur vous les faveurs divines Nous vous accordons de grand coeur la Bénédiction apostolique. Cette Bénédiction s'étend, selon votre désir, non seulement à vous-mêmes qui êtes ici présents, mais également à vos familles, à tous vos collègues empêchés de se trouver eux aussi réunis aujourd'hui au milieu de vous et à tant d'autres qui, s'ils jouissaient de leur liberté civile, se seraient certainement unis à votre manifestation d'hommage filial et dévoué.

\section{LA DECLARATION DE PRINCIPES DE LA C.T.C.C.}

\section{Caractère et but de la C.T.C.C.}

La Confédération des Travailleurs Catholiques du Canada est une organisation syndicale démocratique et libre. Elle est nationale et elle s'inspire dans sa pensée et son action de la doctrine sociale de l'Eglise. Elle croit au rôle primordial des forces spirituelles dans l'établissement et le maintien de l'ordre social.

Elle a pour but de promouvoir les intérêts professionnels, économiques, sociaux et moraux des travailleurs du Canada. Dans sa sphère propre, et en collaboration avec les autres institutions, elle cherche à instaurer pour les travailleurs des conditions économiques et sociales telles qu'ils puissent vivre d'une façon humaine et chrétienne. Elle veut contribuer à l'établissement de relations ordonnées entre employeurs et employés selon la vérité, la justice et la charité. Parmi ses objectifs immédiats, dans ce domaine, elle veut assurer le plein exercice du droit d'association et elle préconise les conventions collectives, les mesures de sécurité sociale et une saine législation du travail. Elle attache également beaucoup d'importance à la formation économique, professionnelle, sociale, intellectuelle et morale des travailleurs. 


\section{Organisation PROFESSIONNELLE}

En vue d'assurer l'harmonie dans les relations de travail et de pourvoir aux besoins de la communauté entière, la C.T.C.C. croit à la nécessité d'établir pour l'économie sociale un statut juridique fondé sur la communauté de responsabilités entre tous ceux qui prennent part à la production.

Le syndicalisme restera toujours pour les travailleurs un moyen essentiel de défendre et de promouvoir leurs intérêts professionnels. $\mathrm{Si}$ le syndicat représente réellement les travailleurs concernés, il est l'organisme normal de négociations, de eprésentation, de collaboration et de participation sur tous les plans: l'entreprise, la profession et l'économie nationale. En conséquence, on doit reconnaître à tous les travailleurs sans distinction, le droit d'association et leur en garantir le libre exercice.

\section{Personne humaine et bien commun}

Le régime économique actuel prédominant dans notre pays déprécie les valeurs humaines et spirituelles. Suivant l'inspiration de ce régime, la recherche de l'intérêt individuel procurerait automatiquement le bien général. Il s'ensuit le mépris de la dignité de l'homme, des légitimes aspirations de la personne humaine et du bien général.

L'accumulation des richesses et la concentration du pouvoir économique entre les mains d'un petit nombre au détriment du bien commun ont été la conséquence d'un système qui s'est donné comme mobile primordial le profit.

C'est un devoir pour chaque citoyen de contribuer au bien commun selon les exigences de la justice sociale. D'autre part, la société est faite pour la personne humaine. Aussi faut-il que la production des biens matériels soit ordonnée à la satisfaction des besoins humains légitimes et que toutes les conditions matérielles, sociales et culturelles favorisent l'épanouissement de chaque travailleur et de sa famille en toute sécurité et liberté.

La C.T.C.C. croit à la dignité primordiale de la personne humaine et à l'égalité fondamentale de tous les êtres humains. Elle n'admet pas qu'on applique un traitement injuste à cause de la langue, de la nationalité, de la race, du sexe et de la religion.

\section{DÉmocratie}

La C.T.C.C. a foi dans la vraie démocratie politique, parce que c'est le système qui garantit le mieux la liberté des citoyens et leur participation aux responsabilités civiles. Elle est d'avis que notre régime démocratique ne doit pas être à la merci de quelques privilégiés qui se servent du pouvoir pour la protection de leurs privilèges et de leurs intérêts égoïstes. La C.T.C.C. croit qu'une véritable démocratie politique ne peut se concevoir sans la démocratisation de l'économie. Elle s'oppose à toute forme de totalitarisme et d'étatisme.

\section{L'Etat}

L'Etat doit promouvoir le bien commun et la C.T.C.C. croit qu'il doit, par ses lois et leur saine application, sauvegarder les droits de chacun et favoriser le développement de groupements intermédiaires autonomes dont la contribution active est nécessaire au maintien de la paix sociale.

\section{Proprí́té PRIVÉE}

La C.T.C.C. reconnaît la légitimité du droit de propriété privée et affirme son double caractère individuel et social; cependant, elle se garde bien d'identifier 
propriété privée et capitalisme. Elle répudie le capitalisme libéral et rejette le marxisme sous toutes ses formes.

L'exercice du droit de propriété doit être réglé selon la nature de l'objet, suivant qu'il s'agit d'un bien d'usage personnel ou d'un bien de production. La propriété privée des biens de production, qui doit rester la règle générale, est grevée de charges sociales plus grandes qui découlent de la nature de ces biens, de leur subordination au bien commun de la société, et du fait que la vie des travailleurs est engagée dans l'entreprise.

L'Etat doit surveiller toute l'activité économique pour assurer la primauté de l'intérêt général sur l'intérêt particulier. Certaines entreprises, à cause de leur grande importance pour le bien-être des citoyens, ou de leur tendance aux abus, ont besoin d'ête suivies de plus près et contenues dans des limites justes par des interventions appropriées.

S'il y a danger pour le bien commun de laisser sous le contrôle d'intérêts privés certains services ou moyens de production, la collectivité doit en assumer la charge. La gestion de ces entreprises sera confiée, autant que possible, à des corps autonomes représentatifs de tous les intéressés.

\section{Collaboration entre les agents de la production}

La vie économique doit être organisée de façon à assurer une collaboration étroite entre les principaux agents de la production et de la distribution des biens. Cette collaboration doit s'établir sur le plan de l'entreprise, de la profession et de l'économie en général.

La C.T.C.C. reconnaît qu'il peut exister plusieurs formes d'entreprises. Elle favorise celles qui en poursuivant leurs fins propres, respectent la personne humaine et servent le mieux le bien commun.

Dans l'entreprise où les uns fournissent le capital et les autres le travail, afin d'intégrer davantage les travailleurs et les intéresser à sa vie, on devrait s'orienter graduellement et dans les limites permises par les circonstances, vers l'introduction d'éléments du contrat de société dans le contrat de travail; la direction des entreprises doit cesser de représenter exclusivement les intérêts du capital.

Au niveau de la profession, les travailleurs et leurs employeurs, par leurs organisations syndicales, doivent se rejoindre en formant des institutions paritaires, qui auront pour but de réglementer la vie professionnelle de telle façon qu'elle serve le mieux possible les intérêts de ses membres et de la société.

A l'échelle provinciale ou nationale, selon les juridictions établies, la C.T.C.C. propose la formation d'organismes appropriés où les représentants des travailleurs et des employeurs seront désignés par les organisations professionnelles intéressées et dont le rôle sera de coordonner et d'orienter la vie économique sous la surveillance de l'Etat.

Enfin, la C.T.C.C. croit que le Canada, de concert avec les autres Etats, doit viser à l'organisation internationale de l'économie afin d'assurer une meilleure distribution des richesses et de garantir en même temps, par la sécurité et la stabilité économique dans le monde, la paix et lharmonie entre les nations.

\section{La famille}

La famille a une telle importance qu'on doit tout faire pour préserver son intégrité, garantir ses droits et assurer son plein épanouissement.

Antérieure à la société civile, dont elle est la première cellule, elle ne peut en aucune façon lui sacrifier son rôle, ses fonctions et ses prérogatives essentielles: 
le droit des époux à une vie conjugale normale, le droit du père de famille à pourvoir à la subsistance des siens, le droit de la mère à accomplir au foyer sa tâche de gardienne, de ménagère et d'éducatrice; le droit des parents à élever leurs enfants et à leur assurer une instruction et une éducation dont ils gardent le contrôle; le droit à une habitation salubre et suffisamment spacieuse dont ils seront, autant que possible, propriétaires.

\section{Droit du Travall}

La société doit assurer à chacun la possibilité de se procurer un emploi stable et rémunérateur, conforme à ses goûts et à ses aptitudes.

\section{Conditions de travail}

Les conditions de travail doivent être saines tant au point de vue moral que physique. Elles doivent laisser au travailleur des loisirs suffisants pour remplir ses devoirs religieux, vivre à son foyer, participer à la vie sociale, se cultiver et se reposer.

\section{RÉmunÉration du TRAVAIL}

La rémunération du travail doit au moins être suffisante pour satisfaire aux besoins normaux de la famille.

Elle doit tenir compte, en plus, de la nature du travail, de la compétence professionnelle, de la productivité, de la situation de l'entreprise, et des exigences du bien commun.

La C.T.C.C. estime qu'il doit y avoir égalité de rémunération entre la maind'oeuvre masculine et la main-d'oeuvre féminine pour un travail de valeur égale.

\section{SÉcurité SOCIALE}

L'insécurité est l'une des caractéristiques de la condition actuelle du travailleur et de sa famille. Elle résulte de l'insuffisance du revenu, de l'instabilité de l'emploi, et d'un manque de protection efficace contre les risques du travail et certains risques inhérents à la vie.

Pour corriger cette situation, la C.T.C.C. croit que l'on doit d'abord ordonner la production des biens matériels à la satisfaction des besoins humains légitimes et favoriser l'établissement de mesures de sécursité sociale telles qu'une politique de plein emploi, des revenus de remplacement et de complément. Ces mesures devront respecter les droits, l'initiative et les prérogatives de chaque citoyen.

\section{MOUVEMENT COOPÉRATE}

La C.T.C.C. voit dans le mouvement coopératif un excellent moyen d'assairissement économique et social et un complément nécessaire à l'action syndicale pour réduire le coût de la vie, humaniser et démocratiser l'économie.

\section{EPARGNe}

L'épargne est un acte de prévoyance et une garantie contre l'insécurité. La C.T.C.C. en reconnaît la nécessité et réclame pour le travailleur la possibilité d'épargner. Elle entend mettre en oeuvre tous les moyens nécessaires à cette fin.

Elle voit dans les coopératives d'épargne et de crédit et certains autres organismes de même nature, des institutions aptes à faire servir l'argent des travailleurs à leur promotion. La plupart des grandes entreprises financières drainent actuelle- 
ment la plus grande partie de l'épargne et contribuent au maintien de la dictature économique.

\section{INSTRUCTION ET CULTURE}

Trop souvent les conditions économiques dans lesquelles vit la famille ouvrière fcnt obstacle à la formation technique, économique, sociale et politique des travailleurs, de même qu'à leur participation à la vie culturelle. L'instruction à tous les degrés et la culture doivent être accessibles aux travailleurs.

\section{FAITS ET COMMENTAIRES}

- Monsieur Jacques Gagnon, directeur du personnel à l'Aluminum Company of Canada Limited, Arvida, poursuit une série de cours aux étudiants du département des Relations industrielles.

- Mgr Pietro Pavan, vice-président des Semaines Sociales d'Italie, professeur aux Universités Angelicum, Gregorium et Latran, a donné une série de cours sur le syndicalisme.

- Monsieur H.J. Clawson, directeur des relations industrielles à la Steel Company of Canada, Limited, Hamilton et Monsieur L. Hemsworth, gérant des relations industrielles à la Canadian Industries Limited, sont également professeurs visiteurs au Département des Relations industrielles.

- Brother Justin, F.S.C., directeur du Département des relations de travail et professeur en relations industrielles au Manhattan College de New York,

- Monsieur Gérard Picard, président général de la Confédération des Travailleurs Catholiques du Canada,

- Monsieur Daniel Leet Shields, ingénieur industriel de l'American Associated Consultants, ingénieurs industriels consultants de New-York et Pittsburg,

- Monsieur Emile Coornhart, professeur au Collège de France sont venus recontrer les étudiants de relations industrielles au cours des trois derniers mois et les ont entretenus de divers problèmes et questions de relations industrielles.

- Monsieur Gérard Dion, ptre, directeur-adjoint du Département des Relations Industrielles, professeur à l'Úniversité Laval a prononcé une conférence aux Marchands détaillarts de Chicoutimi et à la Chambre de Commerce des Jeunes de Rouyn.

- Monsieur Emile Gosselin, secrétaire du Département des Relations Industrielles, professeur à l'Université Laval, a été conférencier invité du Richelieu à Rouyn et de la Ligue des propriétaires du Québec.

- Le Département des Relations Industrielles a participé pendant trois semaines à un Collège ouvrier organisé par les Métallos-Unis d'Amérique dans la région Abitibi-Rouyn-Noranda et a également participé au Collège ouvrier organisé par le Syndicat National d'Aluminium, Arvida. 